\title{
Safety Research of Ultralow Temperature Refrigerator Ship for Seaworthiness
}

\author{
DONG Liang-xiong \\ School of Maritime and Civil Engineering of ZJOU \\ ZheJiang Ocean University \\ ZhouShan, China \\ dongliangxiong@163.com \\ XU Hong-xun \\ Zhejiang Zengzhou Shipbuilding Co., Ltd \\ ZhouShan, China \\ Xuhongxun982@126.com
}

\author{
JIANG Bo \\ Zhejiang Zengzhou Shipbuilding Co., Ltd \\ ZhouShan, China \\ Jiangbo1846@126.com
}

\author{
Zhu Fa-xin \\ School of Maritime and Civil Engineering ofZJOU \\ ZheJiang Ocean University \\ ZhouShan, China \\ zhufax7758@163.com
}

\begin{abstract}
With the rise of the refrigerator ship as a new technology, it is imperative for safety monitoring using the scientific method. In this paper seaworthiness evaluation is employed for safety research. Firstly, according to refrigerator ship characteristics, seaworthiness evaluation content, procedure and its effect factors is analyzed in terms of probability of power loss, and seaworthiness evaluation model is constructed. Then through the analysis of the failure mode of refrigerator ship, two reliability block diagram(RBD) of ship both in refrigerator section and in noload section are proposed. Finally, the model of RBD is transformed to dynamic Bayesian networks(DBN) in which conditional probabilities of nodes are calculated correspondingly. The studies solved the problems which included dynamic, small sample, depended failure of seaworthy analysis in refrigerator ship. The computed results indicated the probability of ship power loss increase significantly in refrigerator section due to the common cause failure. The validity and advantages of proposed method was demonstrated by the example of a $5600 \mathrm{~m}^{3}$ ultralow temperature refrigeration ocean carrier.
\end{abstract}

Keywords- Safety analysis; Seaworthiness; Dynamic Bayesian; refrigerator ship; Failure correlations .

\section{INTRODUCTION}

Global refrigerated cargo transport is a huge space for development, the refrigerator ship as the main transport services in the next foreseeable period of time must be in short supply. The refrigerator ship, where a refrigeration unit is supplied, is operated in the mode of refrigerated condition and no-load condition bringing better economic benefit for fish(Tuna) processing and refrigerator transporting. However, compared to other ships, the new type of refrigerator ship has more particularities in the aspects of low temperature resistance, loss of electricity, and cargo damage incidents occurring more and more in the refrigerated cargo transport. The immaturity of technology and management led to a lot of potential safety hazard existed in the ship operation, and further influenced the safety and efficiency of ship, that is seaworthiness.
The signification of the seaworthiness, an old concept, has changed continuously with the improving attention of the governments and international organizations, the advancements of the relations sciences, and the improvements of ability of shipbuilding [1]. The shipsurvey and the ship safety check is the main means that the administrative departments control and manage ship technical states. In European countries, refrigerator ship applications always be advanced than other countries. Right now in china, the refrigerator ship applied mainly to ocean fishery and international trade which is only in the early stages, the applicative effects remaining to be tested. CCS (China Classification Society) and the Marine Bureau have promulgated specification on the requirements and normalization of the refrigerator ship that have played an instrumental role in design and management of refrigerator ship. The effective safety evaluating and monitoring, especially systematic analysis from the perspective of ship seaworthiness, is need to and can be applied. ZHANG Guang-fa [2] analyzes the criterion of seaworthiness in many ways: voyage area, cargo, weather, and the performance of fishing vessels different from others. WANG Wen-xin [3] have described the phenomenon of engine unstable operation, black out, power drop, high fuel consumption, and have analyzed the common cause failure of refrigeration unit. These analyses are certainly applicable but lack the seaworthiness analyzing from the point of Navigation function. Considering the abovementioned problems, the failure feature of refrigerator ship is comprehensive analyzed, and Bayesian network on the basis of reliability block diagram is built in this paper. The model have good feasibility toward the safety evaluation of refrigerator ship [4].

\section{PROCEED OF SEAWORTHINESS EVALUATION}

There are many ways to deal with safety assessment of complex systems which have process variables and characteristics. Among several techniques available to quantify the occurrence probability of accident scenarios 
or to estimate the failure probability of systems in the context of quantitative risk assessment, probabilistic safety analysis and reliability engineering, the reliability block diagram (RBD) method is the most recognized and widely used [5]. RBD is a deductive, user-friendly methodology constructed intuitively, expressing failure logic and reliable by each connected block. RBD can also be analyzed using well-established algorithms such as binary decision diagrams or analytical methods such as minimal cut sets. However, conventional RBD are characterized by limitations constraining their application in complex dynamic systems where, for instance, redundant failures, non-coherence, polymorphism, multiple distributions, small sample, dependent failure are common. Many scholar have improved the way of RBD, but most of them are restricted on the theory, unable to overcome the fundamental defect of the current practice.

Bayesian networks(BN), who developed in the last century and focused by experts of many domains, is famed for its mathematical foundations and powerful modeling and analysis abilities including making use of all kinds of information. With mature software support, Bayesian network have ability to describe the uncertainly, polymorphism logic relations among dependent failure, thus the BN bring us new idea of logical modeling of ship seaworthiness. This paper propose a model of Bayesian networks expressing the seaworthiness based on RBD, then network condition probability is calculated to determine if ship has ability to navigate safely during day and night. The above proceed is illustrated as the fig1:

\section{SEAWORTHINESS EVALUATION MODELING}

\section{A. Reliability block diagram modeling}

There are multiple mode when refrigerator ship running, accordingly there are multiple RBD corresponding to their system function and analysis intention. For comparison's sake, this paper choose two section, that is refrigerate section and no-load section, of refrigerated ship to analyze. According to the specification requirements from CCS and the Marine Bureau, the RBD of the two section are designed as fig. 2 and fig. 3 . In these diagram the key equipment and system related to safe navigation is chosen as the function block.

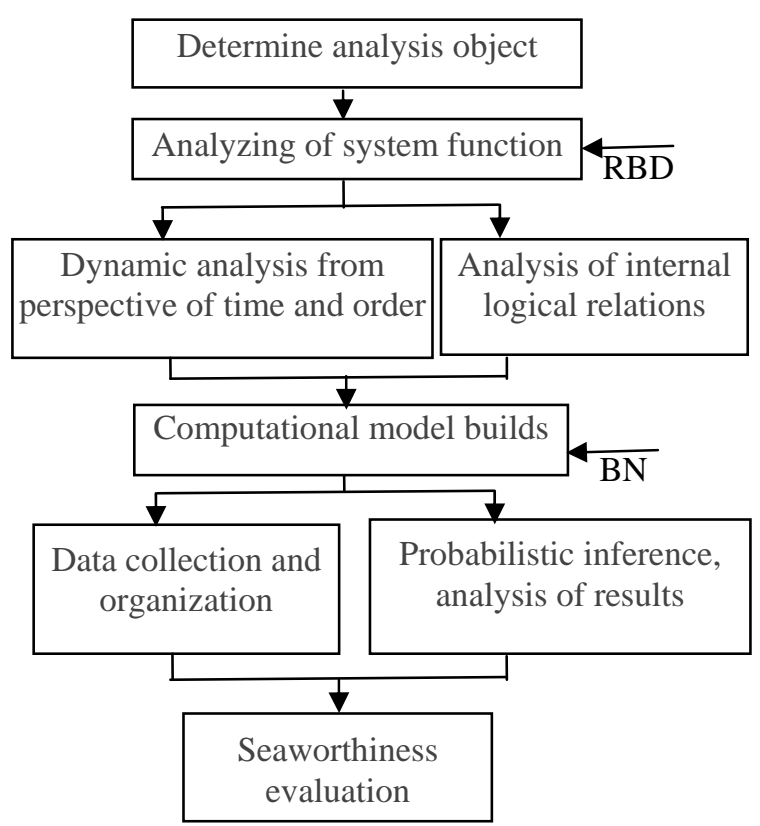

Figure 1. Evaluation flow of ship's seaworthiness

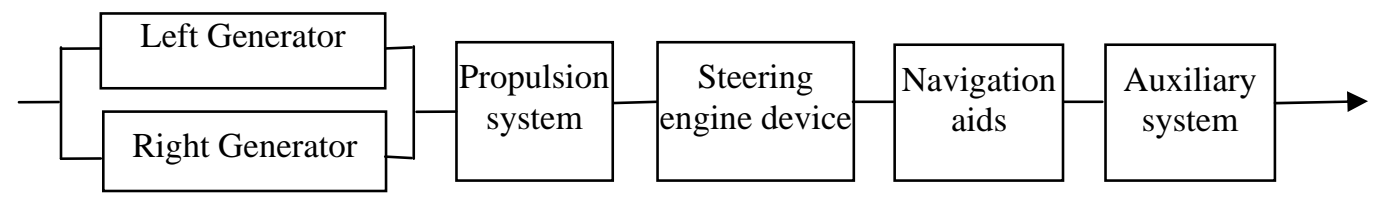

Figure 2. RBD of ship in refrigerated case

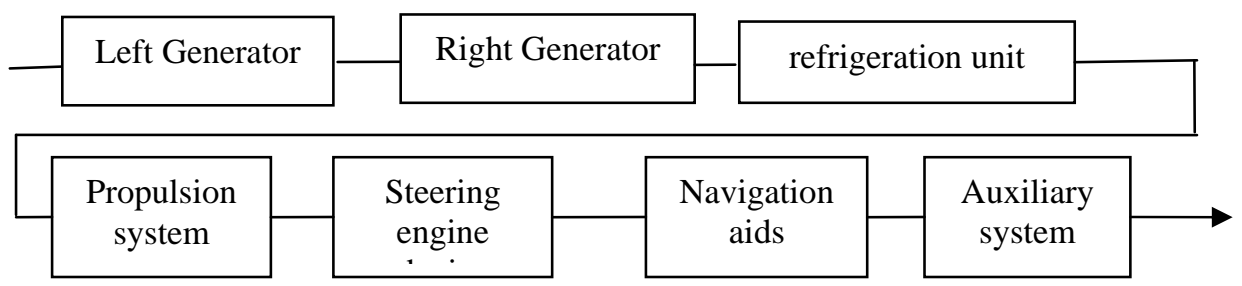

Figure 3. RBD of ship in no-load case

As the fig.2 and fig. 3 shows, the RBD gives the reason of the accidents that happened during the refrigerated cargo transportation as following: generators; propulsion system; steering engine; navigation aids; auxiliary system, etc. In the refrigerating condition, the left generator and the right generator are in series connection because using only one generator cannot maintain enough power for ship voyage; instead, in the no-load condition two generators is in parallel - that is, single failures of generator will not result in black-out in ship.

\section{B. Model transformation between RBD and $B N$}

$\mathrm{BN}$ is a probabilistic method for reasoning under uncertainty which factorizes the joint probability distribution of a set of variables by considering local dependencies, significantly reducing both the system complexity and the computational time. When BN model 
are created, because the use of refrigerated system have an effect on ship equipment system, it is called the common cause failure. If ignore that and suppose every part failure is independent of each other, the seaworthiness evaluation would result in deviation and even many mistakes. Because the model of the common cause failure is so complex that it is difficult to find out key thing what influent system working [6], it is necessary to treat the uncertain logic as function units and add it to $\mathrm{BN}$.

On the other hand, marine equipment failure have the property of polymorphism and dynamic that static Bayesian is difficult to describe. Considering the abovementioned problems, in this paper the dynamic Bayesian network is adopted to describe the logical relationship of failure factors, as shown in the fig. 4 .

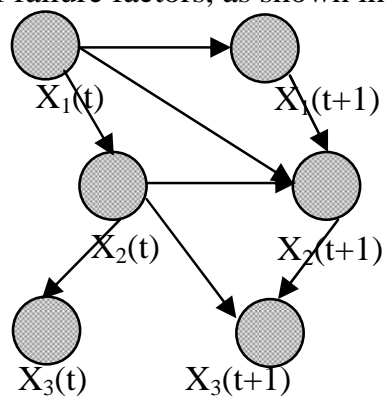

Figure 4. Two temporal slice DBN
In the fig.4, two sections is used to indicate the process of ship performance: one is called initial section ,and the other is called transfer section in which indicate current state variable related to pervious static. Transition probability of state variables in adjacent time will not change over time, which meant that the network have the performance of Markov and time-invariance.

Furthermore, to avoiding the consequent huge structure, DBN is simplified without loss of accuracy when describe the failure mode. There are three network model describing the common failure: explicit model, implicit model, and mixed model. The explicit model, that CHEN Wen-yin [7] have used, have strongly extendibility to indicate the influence of fictitious element. Accordingly, this paper change and improve the model described in that paper, and build a new network model targeting the failure model of refrigerate and no-load two conditions. The built model of Dynamic Bayesian Network is illustrated as the fig.5.

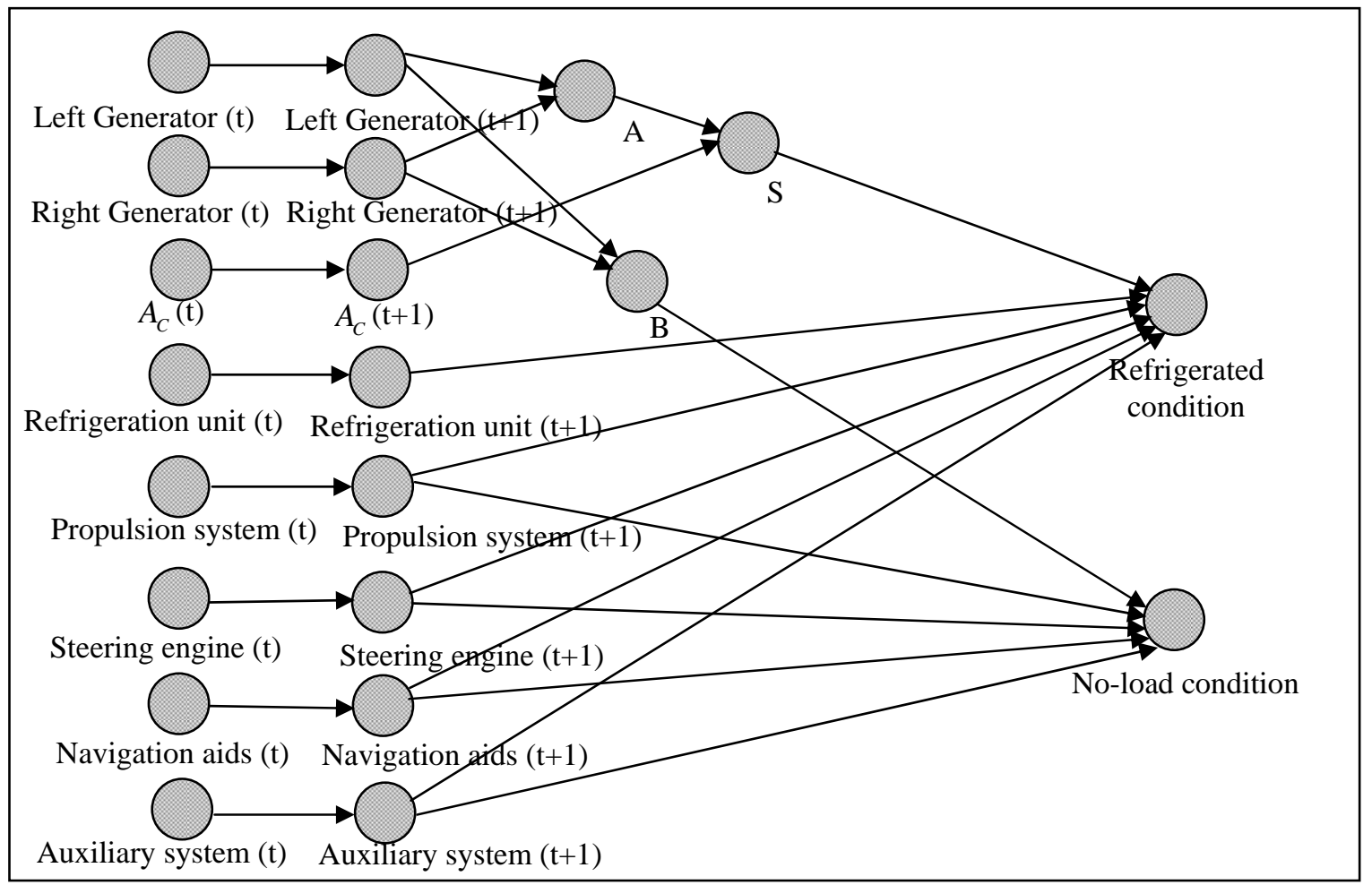

Figure 5. DBN model of two cases of refrigerator ship

\section{Calculate of unite probability distribute}

In the DBN, $B_{1}$ indicate priori network that is defined as unite probability distribute of $X(1)$, in which the priori probability of every point is $P\left(X_{1}\right) ; B_{\rightarrow}$ indicate condition-shift network that is defined as the transfer probability from variable $X(t)$ to variable $X(t+1)$.
Though dynamic Bayesian network usually is semiinfinite network according to variable $X(1), X(2), \ldots, X(\propto)$, we just need consider the most important network according to variable $X(1), X(2), \ldots, X(T)$ within infinite time $(1,2, \cdots, T)$. With any given model the unit probability distribution of $X(1), X(2), \ldots, X(T)$ is: 


$$
\begin{aligned}
& P(X(1), X(2), \ldots, X(T))= \\
& P_{B_{1}}(X(1)) \prod_{t=1}^{T} P_{B_{\rightarrow}}(X(t+1) \mid X(t))
\end{aligned}
$$

$X_{i}(t)$ is used to indicate the NO. $i$ variable whose parent nodes is $\mathrm{Pa}\left(X_{i}(t)\right)$, so the transfer probability from $X(t)$ to $X(t+1)$ is:

$$
\begin{aligned}
& P((X(t+1) \mid X(t))= \\
& \prod_{t=1}^{N} P\left(X_{i}(\mathrm{t}) \mid P a\left(X_{i}(\mathrm{t})\right)\right)
\end{aligned}
$$

So the joint probability distribution of encode from time " 1 " to " $T$ " is:

$$
\begin{aligned}
& P(X(1), X(2), \ldots, X(T))= \\
& \left.\prod_{t=1}^{T} \prod_{i=1}^{N} P X_{i}(t) \mid P a(X(t))\right)
\end{aligned}
$$

\section{Calculate of condition probability}

The functional logic relationship of the system components in RBD provides computation basis for the condition probability of the nodes in $\mathrm{BN}$ [8]. In the refrigerate condition, $A_{1}$ indicate left generator, $A_{2}$ indicate right generator, $A_{c}$ indicate the common cause. $A$ indicate output of engine in series connection, $S$ indicate the actual output. The DBN is illustrated as the fig6, and its condition probability can be calculate by the formula (4)-(7).

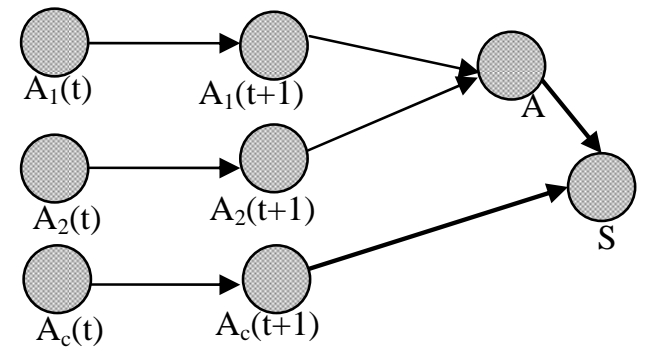

Figure 6. DBN of nodes in series

$$
\begin{aligned}
& P\left(A_{1}(t+1)=1 \mid A_{1}(t)=1\right)=1 \\
& P\left(A_{1}(t+1)=1 \mid A_{1}(t)=1\right)=\int_{t}^{t+1} f_{A_{1}} d t \\
& P\left(A_{2}(t+1)=1 \mid A_{2}(t)=1\right)=1 \\
& P\left(A_{2}(t+1)=1 \mid A_{2}(t)=1\right)=\int_{t}^{t+1} f_{A_{2}} d t
\end{aligned}
$$

$$
\begin{aligned}
& P\left(A_{C}(t+1)=1 \mid A_{C}(t)=1\right)=1 \\
& P\left(A_{C}(t+1)=1 \mid A_{C}(t)=1\right)=\int_{t}^{t+1} f_{A_{C}} d t \\
& P\left(A=1 \mid A_{1}(t+1)=0, A_{2}(t+1)=0\right)=0 \\
& P(A=1 \text { other })=1
\end{aligned}
$$

In the no-load condition, $B$ indicate the output of generator in parallel Do the same calculation, and see that we will get the output of engine in parallel which condition probability can be calculate by the formula followed:

$$
\begin{aligned}
& P\left(B=1 \mid A_{1}(t+1)=1, A_{2}(t+1)=1\right)=1 \\
& P(B=1 \text { other })=0 \\
& \quad P\left(S=1 \mid A=0, A_{C}(t+1)=0\right)=0 \\
& P(S=1 \text { other })=1
\end{aligned}
$$

\section{INSTANCE AND RESULT}

Taken a $5600 \mathrm{~m}^{3}$ ultralow temperature refrigeration ocean carrier for example, the failure rate of ship components, that is the probability of accident taken place, is computed based on ship maintenance record, listing in table 2. The probability of common failure is obtained from the failure of refrigerating system. When the data processing be implemented it is necessary to analyze fault characteristic related to refrigerate system, distinguishing from the ordinary failure and accident, thus the credibility of data would be secure.

TABLE I. FAILURE RATE OF SUBSYSTEMS

\begin{tabular}{|c|c|c|c|}
\hline subsystem & $\lambda\left(h^{-1}\right)$ & subsystem & $\lambda\left(h^{-1}\right)$ \\
\hline $\begin{array}{c}\text { Left } \\
\text { Generator }\end{array}$ & $1 / 14000$ & $\begin{array}{c}\text { Propulsion } \\
\text { system }\end{array}$ & $1 / 10600$ \\
\hline $\begin{array}{c}\text { Right } \\
\text { Generator }\end{array}$ & $1 / 14050$ & $\begin{array}{c}\text { Steering } \\
\text { engine }\end{array}$ & $1 / 9000$ \\
\hline $\begin{array}{c}\text { Refrigeratio } \\
\text { n unit }\end{array}$ & $1 / 8350$ & $\begin{array}{c}\text { Navigation } \\
\text { aids }\end{array}$ & $1 / 7000$ \\
\hline $\begin{array}{c}\text { Refrigeratin } \\
\text { g system }\end{array}$ & $1 / 8450$ & $\begin{array}{c}\text { Auxiliary } \\
\text { system }\end{array}$ & $1 / 7500$ \\
\hline
\end{tabular}

The common numerical simulation method of $\mathrm{BN}$ is multi-branches tree algorithm, junction tree, random sampling method and search method, which can be realized by BayesiaLab or Hugin software, also can be programmed by BNT toolbox of Matlab software [9]. The followed example, using BayesiaLab software, shows that how the probabilities change under two kind of operating modes within $10000 \mathrm{~h}$. 


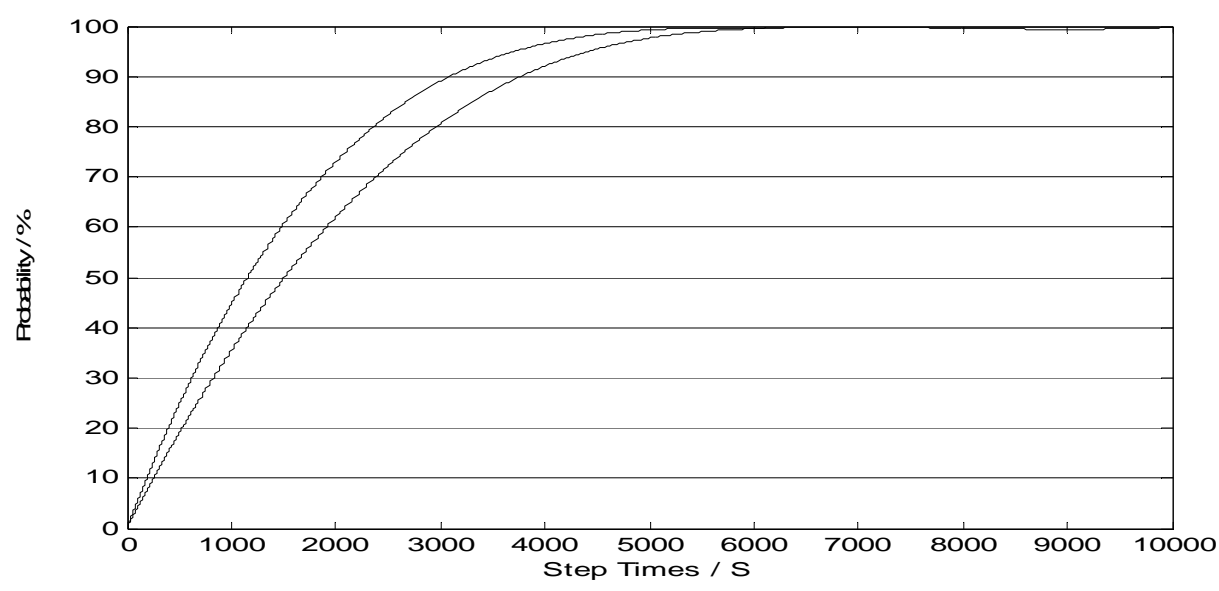

Figure 7. Trend diagram of probability of failure on two cases

The above diagram is plotted by steps times on the horizontal axis and probability on the vertical. The above curve of diagram represent probability of ship failure on refrigerated case and the low curve is that of no-load case. So, at this point the seaworthiness evaluation of refrigerator ship can be finally obtained. Through the safety research based on DBN, the method shows good accordance with the practical circumstance. It is obvious that the change trend of two condition is difference: the probability of failure in refrigerated case is obviously larger than other. The influence of common failure cannot be ignored and precautionary measures should be taken. Refrigerating system, that can decrease the ship seaworthiness at a larger degree than previously assumed, should be especially concerned in the ship safety check [10].

To conclude, during the working of refrigerating system, the dynamics and reliability of refrigerator ship has undergone great changed. The seaworthiness of refrigerator ship takes on the feature of multiple states, non-coherence, failure correlations, and its evaluation become an urgent and complex work. Analyzed the basic failure event during ship voyage, the DBN model is built to effectively express the common caused failure among subsystem and to enhance the reliability of the results. This model, which is used to calculate failure probability, improve the accuracy and reduces the difficulty by effective use of the statistics based on analysis of equipments fault and maintenance law. Result of calculation can give great help in seaworthiness evaluation, safety analysis, and safety decision-making of refrigerator ship.

\section{ACKNOWLEDGMENT}

This work is founded by Zhejiang province major science and technology project, "Study of key technology for $5600 \mathrm{~m}^{3}$ Ultralow Temperature Refrigeration Ocean carrier (2013C03033)", and Zhoushan city scientific and technical projects "research of key technology and industrialization for $5600 \mathrm{~m}^{3}$ ultralow temperature refrigeration ocean carrier(2013C11015)”. Authors would address their thanks to financial aid from scientific research foundation of Zhejiang Ocean University and technical services from Zhejiang Zengzhou Shipbuilding Co., Ltd.

\section{REFERENCES}

[1] IMO. MSC82/INF. 3FSA. Possible improvements on FSA Guidelines, Submitted by Denmark at MSC8[R]. 2006

[2] ZHANG G F, ZHANG Y, YAO J, etc. Development and implementation of safety evaluation system for fishing vessels[J]. Transactions of the Chinese Society of Agricultural Engineering. 2013. 29(17): 137-144.

[3] WANG W X. Research on Safety of Maritime Operation of Refrigerated Cargo [D]. Dalian Maritime University. 2011

[4] DONG Liang-xiong, CHE Hui. Research on Knowledge Acquisition about Condition Identification of Faults in Ship Equipment. The 1st International Conference on Transportation Information and Safety, 2011, Wuhan, China.

[5] HU Shen-ping, FANG Quan-gen,, QIAO Gui-min, et al. Risk Assessment and Risk Control Options of Ship Navigation[J]. Navigation of China, 2006, (3): 34-38.

[6] YI X W, QIAN W X, XIE L Y. Common Cause Failure Model of system Reliability Based on Bayesian Networks[J]. China Mechanical Engineering, 2009: 20(1): 90-94

[7] Chen W Y, Chai J S. Application of the Cause-Consequence Diagram Model for Quantify Grinding Machine System Safety Capability[C]. Proceeding of International Conference on Environmental Pollution and Public Health, Wuhan, China, 2011.

[8] Langseth H, Portinale L. Bayesian Networks in Reliability[J]. Reliability Engineering System Safety, 2006,92: 92-108

[9] HUANG Chang-hai, HU Shen-ping, HAO Yan-ban, et al. Design and Simulation of Read time Dynamic Risk Assessment System for Single Ship Based on FIS Algorithm[J]. Navigation of China, 2011, 34(2):68-73

[10] JIANG W D, LIN S M. Bayesian learning and inference algorithm based on Bayesian Networks Toolbox[J]. Information Technology. 2007(2):5-8. 